\title{
Dynamic Games in Novel Networks: Guest Editors' Forewords
}

\author{
E. Altman ${ }^{1}$ - F. De Pellegrini ${ }^{2}$
}

Published online: 11 May 2016

C) Springer Science+Business Media New York 2016

An increasing number of man-made networked systems, such as social networks and online platforms, govern the interactions between players through predefined actions such as the possibility of sharing informations, accessing a service, express opinions or preferences. Ultimately, the evolution of such systems is determined by strategic interactions developing over complex structures. Strategic interactions, which take place at the local level, e.g., in a node's neighborhood, finally emerge to determine global system configurations. This special issue has collected a set of contributions, which are inspired by this general framework.

In this broad context, two contributions in this special issue are focused on network effects. In particular, coordination games are used as atomic representation of imitation behavior of players which may propagate or not in an interconnected system, e.g., across a social network. Khan et al. study the influence of network topology on the emergence of efficient equilibria in coordination games and explore numerically the convergence time to such configurations. Conversely, Peeters et al. focus on the effect of information diffusion by considering global interaction and confine imitation to the neighborhood of players.

The individual choice of customers of a queuing system is studied in the paper authored by Wiecek and Altman, where the authors develop a theory which captures existence of threshold-type policies in the decision to join or not to join a queue. The authors also study the role of the amount of information about the queue backlog available at customers upon their arrival to the queue.

Shimkin and Altman study a game of timing between a random number of content creators, who compete for position and exposure time over a shared medium such as an online classified list. Contents are ordered according to their submission times, with more recent submissions displayed at the top positions. Content creators have to choose the submission time of their

\footnotetext{
F. De Pellegrini

francesco.depellegrini@create-net.org

E. Altman

eitan.altman@sophia.inria.fr

1 INRIA, Sophia Antipolis, France

2 CREATE-NET, Trento, Italy
} 
contents within a finite time interval, with the goal of each creator to maximize the total expected exposure of this content. The authors analyze the symmetric equilibrium of the game, characterize it in terms of a differential boundary value problem and devise a numerical scheme for its computation.

The role of homophily is studied in the work of Bagagiolo, where the players of a social network tend to align their opinions to the mainstream opinion, i.e., the opinion of the majority. The role of activation costs in order to align to the mainstream opinion is studied through a meanfield game model.

Petrosyan and Sedakov connect the network formation concept to a dynamic cooperative framework: The result of bailing out from a coalition has the meaning of players stopping their activity in the game, removing all the links as well as the ability to establish new ones. The authors hence study the existence of a cooperative solution which is subgame consistent.

Finally, the effect of network heterogeneity on evolutionary dynamics is studied by Zhang et al. The evolution of strategies depends on the neighbors' strategies, and the authors introduce an approximated replicator equation that involves mean value and the variance of the degree distribution. They show that such equation predicts correct behaviors observed in the literature.

We thank the referees for their timely and dedicated work. 